\title{
Análise de jogo no Futebol: Métricas de avaliação do comportamento coletivo
}

\author{
Match analysis on Football: Metrics to evaluate the collective behavior
}

\author{
Filipe Manuel Clemente, ${ }^{1,2^{*}}$ Micael Santos Couceiro, ${ }^{3,4}$ Fernando Manuel Martins,1,5 \\ António José Figueiredo, ${ }^{2,6}$ Rui Sousa Mendes ${ }^{1}$
}

ARTIGO ORIGINAL | ORIGINAL ARTICLE

\begin{abstract}
O jogo de futebol, como realidade complexa, necessita de uma interpretação do comportamento coletivo da equipa. Assim, o presente trabalho objetivou apresentar 4 métricas de avaliação coletiva baseadas na relação espácio-temporal, procurando identificar diferenças nos comportamentos coletivos nos momentos com e sem posse de bola. Para o efeito, analisou-se um jogo de futebol de sete do escalão de formação sub-14. Os resultados evidenciaram diferenças significativas entre os momentos com e sem posse de bola no que se refere ao espaço ocupado pelas equipas $\mathrm{A}\left(F_{(1,1506)}=8.31, p=0.004, \eta^{2}=0.005\right.$, Power $=0.82)$ e $\mathrm{B}\left(F_{(1,1506)}=37.66, p=0.001, \eta^{2}=0.024\right.$, Power $\left.=1.00\right)$, às triangulações efetivas entre jogadores nas equipas $\mathrm{A}\left(F_{(1,1506)}=1343.89, p=0.001, \eta^{2}=0.472\right.$, Power $\left.=1.000\right)$ e $\mathrm{B}\left(F_{(1,1506)}=968.50\right.$, $p=0.001, \eta^{2}=0.391 ;$ Power $\left.=1.00\right)$ e à progressão do centroid nas equipas $\mathrm{A}\left(F_{(1.1506)}=11.79, p=0.001\right.$, $\eta^{2}=0.008$, Power $\left.=0.93\right)$ e B $\left(F_{(1,1506)}=9.43, p=0.001, \eta^{2}=0.006\right.$, Power $\left.=0.87\right)$. Conclui-se com o presente trabalho que as métricas de avaliação coletiva possibilitam uma identificação de relações espácio-temporais entre jogadores, identificando uma expansão maior das equipas na situação com posse de bola, bem como, o avanço do ponto médio da equipa.

Palavras-chave: comportamento coletivo, futebol, análise de jogo, métricas de avaliação
\end{abstract}

ABSTRACT

In the last few years, the collective analysis in football teams has been focused on their complex reality. This study aimed to propose 4 collective metrics based on the spatio-temporal relationship, trying to identify differences between the moments with and without ball possession. A U14 7-a-side football match was analysed. Significant differences were found between the moments with and without ball possession regarding the space covered by team $\mathrm{A}\left(F_{(1,1506)}=8.31, p=0.004, \eta^{2}=0.005\right.$, Power $\left.=0.82\right)$ and $\mathrm{B}\left(F_{(1,1506)}=37.66, p=0.001, \eta^{2}=0.024\right.$, Power $\left.=1.00\right)$, effective area of play on team $\mathrm{A}\left(F_{(1,1506)}=\right.$ $1343.89, p=0.001, \eta^{2}=0.472$, Power $\left.=1.000\right)$ and $B\left(F_{(1,1506)}=968.50, p=0.001, \eta^{2}=0.391 ;\right.$ Power $=$ $1.00)$, as well as at centroid position for team A $\left(F_{(1.1506)}=11.79, p=0.001, \eta^{2}=0.008\right.$, Power $\left.=0.93\right)$ and $\mathrm{B}\left(F_{(1,1506)}=9.43, p=0.001, \eta^{2}=0.006\right.$, Power $\left.=0.87\right)$. As a conclusion, it was possible to analyse the spatio-temporal relationship between teammates, identifying expansion behaviour with ball possession, as well as a centroid progression at the field with the collective metrics selected.

Keywords: collective behavior, football, match analysis, metrics

Artigo recebido a 20.12.2012; $1^{\text {a }}$ Revisão 19.06.2013; Aceite 31.07.2013

${ }^{1}$ Instituto Politécnico de Coimbra, ESEC, DE, Portugal

${ }^{2}$ Faculdade de Ciências do Desporto e Educação Física da Universidade de Coimbra, Portugal

${ }^{3}$ RoboCorp, Instituto Politécnico de Coimbra, ISEC, DEE, Portugal

${ }^{4}$ Ingeniarius, Lda., Portugal

${ }^{5}$ Instituto de Telecomunicações, Delegação da Covilhã, Portugal

${ }^{6}$ CIDAF, Faculdade de Ciências do Desporto e Educação Física da Universidade de Coimbra, Portugal

* Autor correspondente: Faculdade de Ciências do Desporto e Educação Física, Universidade de Coimbra, Estádio Universitário, Pavilhão 3, 3040-156 Coimbra, Portugal; E-mail: filipe.clemente5@gmail.com 


\section{INTRODUÇÃO}

Os desportos de equipa caracterizam-se pela sua realidade dinâmica e complexa resultante da interação permanente entre os seus constituintes (Gréhaigne, Godbout, \& Bouthier, 1999). Nessa perspetiva, uma das principais missões dos técnicos desportivos relaciona-se com a capacidade de sistematizar a dinâmica funcional da sua equipa, procurando potenciar as relações entre jogadores no sentido de melhorar a performance coletiva (Davids, Button, \& Bennett, 2008). Assim, a análise de jogo tem vindo a desenvolver o seu potencial integrador no sentido de dotar os treinadores de informações pertinentes que fundamentem as opções de treino e adoção de estratégias de jogo (Carling, Williams, \& Reilly, 2005).

Comummente a análise de jogo tem vindo a basear-se na análise notacional de jogo, registando as ações técnicas predominantes, bem como, na análise sequencial procurando analisar os comportamentos táticos dos jogadores (Costa, Garganta, Greco, Mesquita, \& Seabra, 2010). Ambas as análises são indubitavelmente pertinentes para a constituição de um entendimento holístico sobre a realidade do jogo.

Complementarmente, novos sistemas de deteção automática de jogadores, que identificam o posicionamento do jogador num plano cartesiano, têm possibilitado a inspeção cinemática da ação dos jogadores (Carling, Bloomfield, Nelsen, \& Reilly, 2008). Apesar de todos os sistemas enunciados, novas métricas de avaliação complementares aos registos observacionais têm surgido nos últimos anos, recorrendo aos avanços na deteção automática de jogadores (Bartlett, Button, Robins, DuttMazumder, \& Kennedy, 2012).

De entre as métricas baseadas no posicionamento cartesiano dos jogadores, as mais utilizadas são: i) centroid (Frencken, Lemmink, Delleman, \& Visscher, 2011); ii) índice de dispersão (Bourbousson, Sève, \& McGarry, 2010); iii) índice de extensão (Moura, Martins, Anido, Barros, \& Cunha, 2012); iv) área coberta (Okihara et al., 2004); v) área efetiva de jogo (Clemente, Couceiro, \& Martins, 2012).
O centroid baseia-se no cálculo do ponto médio ponderado relativamente à distribuição de todos os jogadores da equipa (Frencken et al., 2011). Recorrendo ao conceito de centroid, o índice de dispersão desenvolve-se a partir da soma das distâncias dos jogadores em relação ao ponto médio da equipa (Bourbousson et al., 2010). Semelhante conceito é apresentado por Moura et al (2012) através da métrica de índice de extensão. Quanto à área coberta baseia-se na determinação das triangulações dos jogadores da equipa (excluindo o guarda-redes), computando a soma das áreas das mesmas (Frencken et al., 2011). A área efetiva de jogo fundamenta-se na área coberta, mas determinando um distanciamento máximo entre jogadores na fase defensiva para formar os triângulos eficazes (Clemente et al., 2013). Refira-se que as métricas de avaliação do comportamento coletivo não substituem a análise notacional e/ou sequencial. No entanto, conseguem mensurar com elevado grau de validade e fiabilidade o distanciamento dos jogadores no campo, determinando indiretamente alguns comportamentos de auto-organização incorporados numa visão integrada dos sistemas dinâmicos (Duarte, Araújo, Correia, \& Davids, 2012).

No futebol, bem como, em modalidades coletivas existem fatores contextuais como o resultado de jogo, o local de jogo, a classificação geral das equipas no campeonato ou o estado de posse de bola que determinam a alteração de comportamentos técnico-táticos (Lago \& Martín, 2007). De entre todos os fatores possíveis, o estado de posse de bola determina o comportamento entre jogadores da própria equipa no sentido de se autorregularem em função de um objetivo comum. No estado de não posse de bola o objetivo dos jogadores passa por se auto-organizarem procurando reduzir espaços possíveis de penetração e visando a recuperação da bola no menor período de tempo possível evitando o ataque adversário (Costa, Garganta, Greco, \& Mesquita, 2009). Pelo contrário, no estado de posse de bola, o objetivo passa por facultar ao portador de bola opções para prosseguir o processo 
ofensivo com eficácia, objetivando a finalização (Castelo, 1996). Apesar da literatura no âmbito da análise notacional e sequencial ter desenvolvido investigações no sentido de identificar diferenças de comportamentos técnico-táticos a partir do estado de posse de bola (Lago, 2009), o facto é que até agora nenhuma métrica de avaliação espácio-temporal considerou este fator para a sua análise. De facto, a maioria dos estudos reportados enfatizam o relacionamento espácio-temporal no âmbito da análise sistémica, não considerando o estado de posse de bola. Diferenciadamente da análise notacional ou observacional, a maioria das métricas desenvolvidas atualmente no âmbito da análise de jogo não consideram a posse de bola, apenas consideram o posicionamento bidimensional dos jogadores e analisam os golos sofridos ou marcados em função de uma análise fragmentada do jogo e não contínua ao contrário do proposto no presente trabalho. Desta forma, não considerando a posse de bola, o conhecimento sobre o relacionamento espácio-temporal entre jogadores encontra-se ainda em fase de evolução pelo que afigura-se pertinente atualizar algumas métricas computacionais de análise para propiciar aos técnicos desportivos uma possibilidade de interpretação sobre as diferenças de comportamento entre os estados de posse e sem posse de bola ao longo do jogo e de forma contínua e não fragmentada. Este afigura-se portanto como o caráter inovador do presente artigo, não se pretendo fazer uma comparação com os resultados proveniente de outros métodos de análise, pois na realidade, os mesmos não são passíveis de serem comparadas pelas suas diferenças contextuais de base.

É a partir desta oportunidade que se pretende com o presente estudo analisar a organização coletiva dos jogadores nos momentos com e sem posse de bola, utilizando métricas de avaliação. Para o efeito utilizar-se-ão os métodos de centroid ponderado para verificar a localização média no terreno de jogo, o índice de dispersão ponderado e área coberta para análise da concentração defensiva e espaço ofensivo, bem como, a área efetiva de jogo com o objetivo de analisar a cobertura ofensiva e defensiva.

Para diferenciar o momento ofensivo e defensivo das equipas, considerar-se-á o estado de posse de bola em cada instante. Será expectável, considerando os pressupostos de cada princípio de jogo que, na fase defensiva (i.e., sem posse de bola), a equipa se aproxime da sua baliza, reduzindo a dispersão no campo e aumentando a proximidade entre jogadores. Inversamente, no processo ofensivo (i.e., com posse de bola), será expectável a maior aproximação da equipa da baliza adversária, aumentando a dispersão dos jogadores pelo terreno de jogo, bem como, a área ocupada.

\section{Participantes}

\section{MÉTODO}

Analisou-se um jogo oficial de sub-14 referente à final da competição distrital. Os jogadores das equipas "A" e "B" foram alvos de filmagem durante todo $\mathrm{o}$ jogo para posterior rastreamento posicional. O estudo foi conduzido cumprindo as normas éticas de tratamento de humanos e animais ao abrigo do protocolo de Helsínquia.

\section{Procedimentos de Recolha de Dados}

A filmagem do jogo concretizou-se através da utilização da câmara de filmagem Gopro Hero com resolução de $1280 \times 960$, com capacidade de processamento de imagem em $30 \mathrm{~Hz}$ (30 frames por segundo). A câmara foi colocada num patamar superior, de forma a abranger todo o campo sem necessidade de a movimentar. O campo foi alvo de calibração, utilizando marcadores espaciais que possibilitassem a compatibilização com métodos de conversão posicional.

O primeiro passo para a coleta dos dados consistiu na gravação do comportamento dos jogadores usando a câmara de filmagem GoPro Hero (descrita anteriormente). No estudo da situação de jogo de $7 \times 7$ a câmara de filmagem foi colocada numa plataforma centrada e elevada 10 metros de altura e 5 metros de 


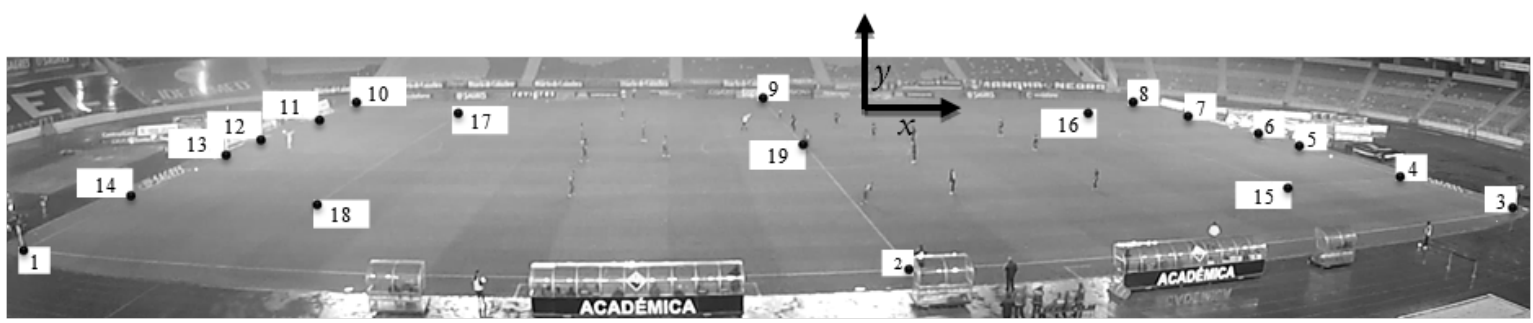

Figura 1. Calibração inicial para extração dos coeficientes DLT com base nas referências reais da Tabela 1

afastamento em relação à linha de fundo do campo. Esta centralidade da câmara e considerando que a mesma filma com uma angulação de $180^{\circ}$ permitiu capturar todo o espaço de prático sem necessidade de alterar o seu posicionamento ao longo do jogo. O espaço de prática foi calibrado com 19 pontos de identificação nas linhas conhecidas do campo regulamentar. Esses pontos de calibração foram identificados metricamente a partir do ponto zero de referência que se encontrava no centro do campo de jogo. Desta forma, valores positivos e negativos foram considerados em função do posicionamento do referencial. No presente estudo, os valores negativos no eixo longitudinal $(x)$ referiam-se à metade do campo de jogo mais próxima na filmagem, sendo que a metade oposta do campo se referia a valores positivos. Quanto ao eixo lateral $(y)$ os valores negativos relacionavam-se com a metade do espaço do lado esquerdo do campo de jogo e os valores positivos referiam-se ao lado direito do campo.

Após a filmagem, o espaço físico foi calibrado usando a técnica das transformadas diretas lineares, Direct Linear Transformation (DLT), consistindo na equivalência proporcional do espaço virtual para o espaço físico real (AbdelAziz, \& Karara, 1971). Esta calibração baseiase na identificação dos marcadores do campo (coordenadas reais) na imagem virtual (coordenadas virtuais) (Fernandes et al., 2010). Para efetuar este procedimento recorreu-se ao software informático MatLab. Numa primeira fase efetuou-se a calibração com base na primeira frame de cada jogo. O processo de calibração inicial teve como objetivo a extração dos coefi- cientes DLT $^{1}$ provenientes do mapeamento entre 19 pontos bidimensionais no espaço virtual (pixéis) para o espaço físico real (metros) seguindo a correspondência entre a Figura 1 e a Tabela 1.

\section{Tabela 1}

Correspondência entre espaço virtual (pixéis) representado na Figura 1 e espaço físico (metros) para efeitos de calibração inicial

\begin{tabular}{crr}
\hline \multirow{2}{*}{ Ponto } & \multicolumn{2}{c}{ Espaço físico real [metros] } \\
& \multicolumn{1}{c}{$y$} & \multicolumn{1}{c}{$x$} \\
\hline 1 & 34.15 & -52 \\
2 & 34.15 & 0 \\
3 & 34.15 & 52 \\
4 & 20.25 & 52 \\
5 & 3.75 & 52 \\
6 & -3.75 & 52 \\
7 & -20.25 & 52 \\
8 & -34.15 & 52 \\
9 & -34.15 & 0 \\
10 & -34.15 & -52 \\
11 & -20.25 & -52 \\
\hline
\end{tabular}

De seguida, desenvolveu-se um interface gráfico permitindo visualizar uma frame do jogo a cada segundo. Durante cada frame era exigido ao utilizador identificar as localizações de todos os jogadores e da bola seguindo a abordagem típica do point and click. Essa identificação correspondia a um ponto na imagem centrado com o posicionamento dos pés dos jogadores. Por sua vez, cada ponto no espaço vir-

\footnotetext{
${ }^{1} \mathrm{O}$ script que permite o cálculo dos coeficientes DLT encontra-se disponível em http://isbweb. org/software/movanal/dltfu2d.m
} 

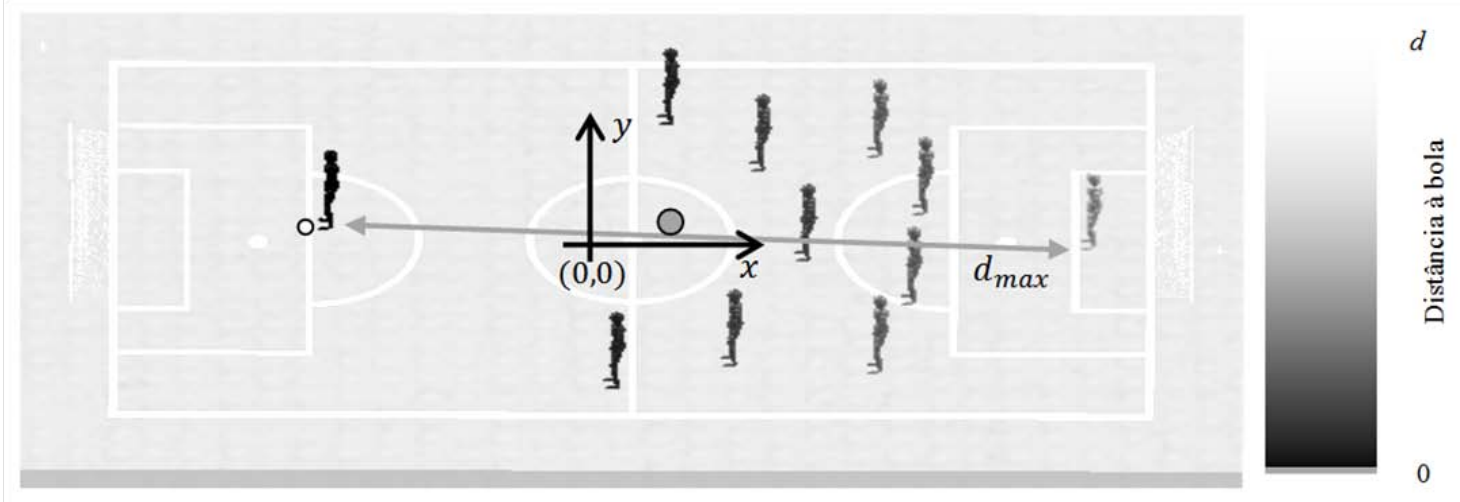

Figura 2. Centroid da equipa

tido algoritmicamente ${ }^{2}$ em função da relação entre as coordenadas virtuais e as coordenadas reais definidas na Figura 1 e Tabela 1.

De forma a garantir a fiabilidade da conversão definiram-se testes de experiência com pontos aleatórios no campo previamente recolhidos no campo com coordenadas reais e medidos metricamente no espaço. Posteriormente, na plataforma desenvolvida computacionalmente identificaram-se os pontos de teste e os resultados permitiram identificar que o maior desvio de erro foi de $5 \mathrm{~cm}$ em relação às coordenadas reais. Esta margem de $5 \mathrm{~cm}$ foi considerada viável para efetuar o estudo, no sentido em que não comprometia o principal fundamento do estudo, i.e., identificar o relacionamento espácio-temporal entre jogadores nos momentos com e sem posse de bola. Para uma descrição detalhada da técnica DLT sugere-se a consulta de Woltring e Huiskes (1990).

\section{Desenvolvimento das Métricas de Avaliação Coletiva}

Com a finalidade de desenvolver as métricas de análise, optou-se por adaptar algumas particularidades dos métodos anteriormente estudados (e.g., centroid, índice de dispersão, área coberta e área efetiva de jogo) visando ajustálos aos objetivos de avaliação coletiva.

\footnotetext{
${ }^{2} \mathrm{O}$ script que permite o remapeamento das coordenadas virtuais em reais, tendo como input os coeficientes DLT, encontra-se disponível em http://isbweb.org/software/movanal/reconfu2.m
}

\section{Analisando a progressão no terreno de jogo}

A noção de homogeneidade de relacionamento dos jogadores no que se refere ao jogo e posicionamento da bola é substancialmente importante. Para o efeito, considera-se pertinente analisar o centro médio dos jogadores o qual designar-se-á por centroid ponderado (Clemente et al., 2013). Este método permite verificar o "centro de massa ponderado da equipa", i.e., ponderará o posicionamento dos jogadores em função da bola e atribuirá pesos a cada um dos jogadores, contribuindo assim para o cálculo do centroid da equipa (Figura 2).

A posição the $i^{\text {th }}$ jogador é definido por $\left(x_{i}\right.$, $\left.y_{i}\right)$. A relevância de cada jogador para o centroid da equipa será ponderado, i.e., $w_{i}$ ponderação, poderá basear-se na distância euclidiana (Clemente et al., 2013):

$$
w_{i}=1-\frac{\sqrt{\left(x_{i}-x_{b}\right)^{2}+\left(y_{i}-y_{b}\right)^{2}}}{d_{\max }}
$$

onde $\left(x_{b}, y_{b}\right)$ corresponde à posição da bola $\mathrm{e}$ $d_{\max }$ é a distância euclidiana do jogador mais afastado em relação à bola, em cada iteração.

Analisando o princípio da concentração defensiva e espaço ofensivo

Os princípios da concentração defensiva e espaço ofensivo definem-se intrinsecamente como uma relação inversa em função do estado de posse de bola, designando-se de relação “expansão-contração" (Bartlett et al., 2012). 
Uma forma de analisar esta relação será através da mensuração da distância entre jogadores da mesma equipa, bem como, da área abrangida coletivamente (Moura et al., 2012). Assim, o índice de dispersão ponderado possibilita uma compreensão da dispersão dos jogadores em torno do seu ponto médio coletivo ponderado (i.e., centroid) (Clemente et al., 2013):

$$
S_{\text {ind }}=\frac{\sum_{i=1}^{N} w_{i} d_{i}}{\sum_{i=1}^{N} w_{i}}
$$

onde $d_{i}$ é a distância euclidiana entre o jogador $i$ e o centroid da equipa:

$$
d_{i}=\sqrt{\left(x_{i}-\bar{x}\right)^{2}+\left(y_{i}-\bar{y}\right)^{2}}
$$

Através do índice de dispersão será possível verificar a maior ou menor dispersão dos jogadores em função do seu centro, resultando assim, na interpretação sobre a sua expansão ou contração em função dos momentos com e sem posse de bola.

No entanto, poderá não ser suficiente a análise da dispersão. Assim, propõem-se neste artigo a análise da área ocupada pelos jogadores (Figura 3).

A área coberta poderá ser computada através da soma das triangulações existentes e analisadas na área efetiva, resultando assim, no valor da cobertura total do polígono formado pela equipa (Moura et al., 2012).

Analisando o potencial de cobertura: Triangulações efetivas de jogo

A área efetiva de jogo constitui-se como um método de avaliação baseado nas triangulações constituídas pelos jogadores da equipa (Clemente et al., 2012). O futebol é composto por múltiplas triangulações que objetivam conferir apoio ofensivo ou defensivo ao jogador que intervém de forma direta junto da bola, tanto

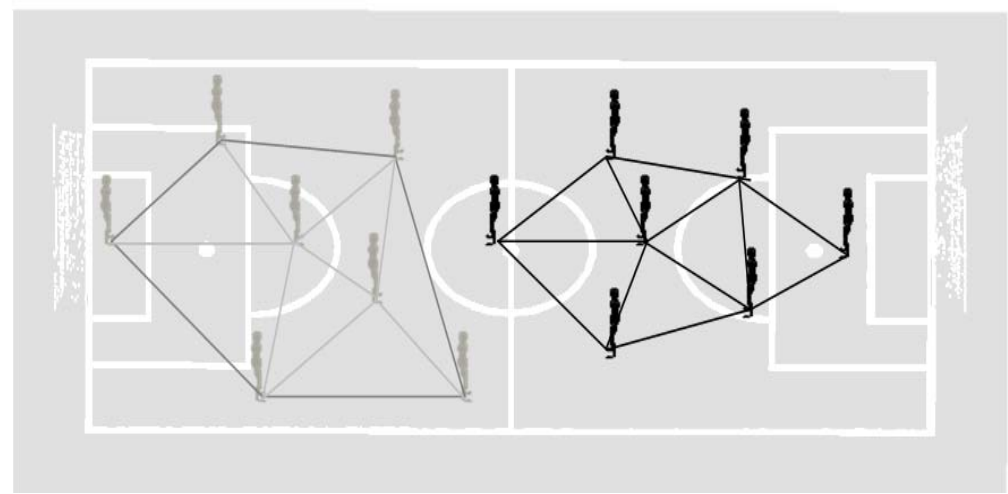

Figura 3. Área coberta por cada equipa

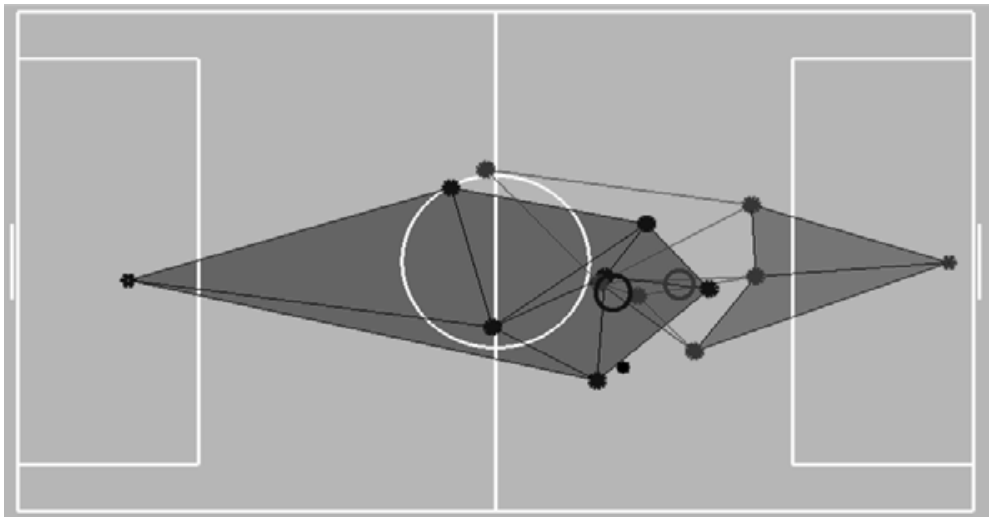

Figura 4. Exemplo de constituição de triangulações eficazes 
como portador da mesma, tanto como defensor direto (Lucchesi, 2001). Assim, importa analisar as triangulações geradas nos momentos com e sem posse de bola (Figura 4). No entanto, as triangulações apenas se tornam efetivas se conferirem potencial à ação (Dooley \& Titz, 2011).

Exemplificando, uma triangulação defensiva apenas se constituirá se os jogadores se manterem próximos entre si, impedindo a reduzindo as possibilidades de penetração adversária (Trapattoni, 1999). Importa salientar que este distanciamento é variável em função das pretensões do treinador, no entanto, alguma literatura aponta o perímetro máximo de 36 metros resultante da soma de 12 metros entre cada membro (Dooley \& Titz, 2011). Assim, nos momentos em que triangulações ofensivas e defensivas se sobreponham, apenas serão consideradas triangulações defensivas eficazes as que possuírem um perímetro inferior a 36 metros (Clemente et al., 2012). No caso de não interceção entre triangulações defensivas e ofensivas todas serão consideradas eficazes. Para o efeito de análise serão considerados todos os segundos de jogo útil, atendendo sempre à equipa com posse de bola.

Importa enaltecer que as triangulações, per se, não garantem a concretização dos princípios táticos de cobertura ofensiva e defensiva. Permite sim, analisar o potencial para o efeito, i.e., a proximidade entre jogadores na fase defensiva permitirá uma maior possibilidade de apoiar o jogador em contenção.

\section{Análise Estatística}

Para a análise estatística dos momentos com e sem posse de bola, utilizou-se o teste estatístico ANOVA one-way, cumpridos os pressupostos de normalidade e homogeneidade da amostra (Maroco, 2010). Adicionalmente, verificou-se a dimensão do efeito dos resultados estatísticos, bem como, o seu poder (Pallant, 2011). Todo o processo estatístico foi concretizado através do software estatístico SPSS, para um nível de significância de 5\%.

\section{RESULTADOS}

Os resultados obtidos comparam o estado com e sem posse de bola para cada equipa (i.e., A e B). Através de tal procedimento será possível comparar os valores entre os estados de posse de bola para cada equipa, i.e., preservando a essência do modelo comportamental de jogo das mesmas (Tabela 2).

\section{Centroid no eixo do x (baliza-a-baliza)}

Existem diferenças estatisticamente significativas entre os estados de posse de bola na equipa $\mathrm{A} \quad\left(F_{(1,1506)}=11.79, p=0.001, \eta^{2}=\right.$ 0.008 , Power $=0.93)$ e na equipa $\mathrm{B}\left(F_{(1,1506)}=\right.$ 9.43, $p=0.001, \eta^{2}=0.006 ;$ Power $\left.=0.87\right) . \mathrm{Em}$ ambos os casos aproximam-se da sua zona defensiva, no momento sem bola.

\section{Centroid no eixo do y (lateral-a-lateral)}

Existem diferenças estatisticamente significativas entre os estados de posse de bola na equipa A $\left(F_{(1,1506)}=19.70, p=0.001, \eta^{2}=\right.$ 0.013 , Power $=0.99)$ e na equipa $\mathrm{B}\left(F_{(1,1506)}=\right.$ 25.11, $p=0.001, \eta^{2}=0.016$, Power $\left.=1.00\right)$.

Tabela 2

Análise descritiva dos métodos aplicados para a análise do comportamento tático

\begin{tabular}{ccccccc}
\hline Equipa & $\begin{array}{c}\text { Posse de Bola } \\
{[\mathrm{BP}]}\end{array}$ & $\begin{array}{c}\text { Centroid } \\
\text { eixo- } x[\mathrm{~m}]\end{array}$ & $\begin{array}{c}\text { Centroid eixo-y } \\
{[\mathrm{m}]}\end{array}$ & $\begin{array}{c}\text { Índice de } \\
\text { Dispersão }[\mathrm{m}]\end{array}$ & Área Coberta $\left[\mathrm{m}^{2}\right]$ & $\begin{array}{c}\text { Área Efetiva } \\
\text { de Jogo }\left[\mathrm{m}^{2}\right]\end{array}$ \\
\hline \multirow{2}{*}{$\mathrm{A}$} & Com BP & $-0.63 \pm 15.93$ & $0.26 \pm 10.11$ & $8.19 \pm 2.27$ & $3783.65 \pm 1489.63$ & $3511.82 \pm 930.91$ \\
& Sem BP & $-3.39 \pm 13.92$ & $-2.06 \pm 9.48$ & $8.29 \pm 2.22$ & $3551.41 \pm 1571.74$ & $1023.63 \pm 1458.40$ \\
\hline \multirow{2}{*}{ B } & Com BP & $1.79 \pm 14.71$ & $-2.58 \pm 8.96$ & $9.01 \pm 2.42$ & $4170.17 \pm 1818.05$ & $3943.03 \pm 1836.41$ \\
& Sem BP & $4.27 \pm 15.53$ & $-0.22 \pm 8.81$ & $8.36 \pm 2.68$ & $3589.61 \pm 1766.91$ & $1457.72 \pm 1261.45$ \\
\hline
\end{tabular}




\section{Índice de Dispersão}

Existem diferenças estatisticamente significativas entre os estados de posse de bola na equipa B $\left(F_{(1,1506)}=22.78, p=0.001, \eta^{2}=\right.$ 0.015 , Power $=1.00)$. Não existem diferenças estatisticamente significativas entre os estados de posse de bola na equipa $\mathrm{A}\left(F_{(1,1506)}=0.60\right.$, $p=0.441, \eta^{2}=0.001$, Power $=0.12$ ).

\section{Área Coberta}

Existem diferenças estatisticamente significativas entre momentos com e sem posse de bola na equipa $\mathrm{A}\left(F_{(1,1506)}=8.31, p=0.004\right.$, $\eta^{2}=0.005$, Power $\left.=0.82\right)$ e equipa $\mathrm{B}\left(F_{(1,1506)}=\right.$ 37.66, $p=0.001, \eta^{2}=0.024$, Power $=1.00$ ).

\section{Área Efetiva de Jogo}

Existem diferenças estatisticamente significativas entre momentos com e sem posse de bola na equipa A $\left(F_{(1,1506)}=1343.89, p=0.001\right.$, $\eta^{2}=0.472$, Power $\left.=1.00\right)$ e B $\left(F_{(1,1506)}=968.50\right.$, $p=0.001, \eta^{2}=0.391$, Power $\left.=1.00\right)$.

\section{DISCUSSÃO}

O treinador necessita de uma interpretação da realidade da equipa e do seu comportamento ao longo do jogo de forma a percecionar os pontos de organização e necessidades de melhoria, permitindo transferir esta avaliação para o treino e reduzir as desregulações comportamentais coletivas (Costa et al., 2010). Desta forma, métricas de avaliação coletiva são uma ferramenta necessariamente importante para o treinador, auxiliando na sua tomada de decisão ao longo do jogo e, fundamentalmente, após o jogo (Clemente et al., 2013).

Assim, este trabalho propôs-se a apresentar métricas de avaliação do comportamento coletivo baseadas no relacionamento espacial entre jogadores, procurando através dos resultados providenciados, analisar diferenças entre os momentos com e sem posse de bola. Considerando que o estado de posse de bola se relaciona diretamente com os momentos defensivos e ofensivos (Lago-Peñas \& Dellal, 2010), foi objetivo do trabalho caracterizar os comportamentos coletivos em ambos os momentos.
Utilizando a métrica da área efetiva de jogo, que analisa as triangulações realizadas entre jogadores (Clemente et al., 2012), bem como o potencial para a cobertura, foi possível constatar que no momento defensivo a equipa reduziu os espaços interpessoais, ocupando uma área inferior efetiva. Este comportamento poderá relacionar-se com a necessidade de reduzir os espaços possíveis de penetração e, sobretudo, formar triangulações defensivas eficazes o que, ao serem cumpridas, irá reduzir necessariamente o espaço ocupado (Dooley \& Titz, 2011). Inversamente, no momento ofensivo, o maior espaço ocupado poderá relacionar-se com a possibilidade de as triangulações ofensivas serem mais dispersas considerando o espaço existente em campo devido ao encurtamento de distâncias da equipa adversária (Castelo, 1996).

Esta noção de afastamento ou redução dos espaços interpessoais poderão ser interpretados considerando a relação contração-expansão entre as equipas ao longo do jogo (Moura et al., 2012). Pressupondo que tal facto poderá ser analisado através da dispersão dos jogadores em torno do seu ponto médio, o índice de dispersão surge como métrica natural para avaliar o cumprimento do princípio tático de concentração defensiva e espaço ofensivo (Bartlett et al., 2012). Desta forma, através do presente trabalho foi possível verificar diferenças estatisticamente significativas na equipa $B$, denotando-se uma maior dispersão entre jogadores no momento defensivo e redução da mesma no momento defensivo. No entanto, esta relação poderá encontrar-se dependente de subprincípios da própria equipa, i.e., padrões de comportamento próprios e específicos da equipa (Clemente et al., 2013). Tal facto poderá justificar a não diferenciação significativa do índice de dispersão da equipa A nos momentos defensivos e ofensivos. Assim, desta análise emerge uma margem de trabalho futuro para que analistas interpretem os resultados atendendo às especificidades próprias da equipa. Efetivamente, tal já acontece com a análise notacional e sequencial, recorrendo a indicado- 
res de posse de bola, número de remates ou número de passes para relacioná-los com ações táticas da equipa (Hughes \& Franks, 2004).

Assim, é compreensível que o centroid no eixo do $y$ (lateral-a-lateral) apresente tendências diferentes para cada equipa (Frencken et al., 2011). Este ponto médio ponderado dos jogadores da equipa (i.e., centroid) representa uma possibilidade de identificar eventuais padrões para a equipa atacar com maior regularidade numa das laterais. Tal facto poderá caracterizar a compreensão de como a equipa se organiza para a construção ofensiva (Bourbousson et al., 2010). A reduzida oscilação no eixo do $x$ (baliza-a-baliza) em função dos momentos ofensivos e defensivos, poderá indicar a capacidade de a equipa manter uma preocupação permanente em preparar os estados de transição defensiva ou ofensiva (Castelo, 1996). No entanto, tais evidências apenas poderão ser confirmadas recorrendo a métodos complementares, tais como a análise sequencial (Costa et al., 2010). No fundo, pretende-se enfatizar que apesar do grande potencial das métricas de avaliação coletiva, o presente desenvolvimento tecnológico ainda não possibilita identificar determinados comportamentos específicos apenas analisados através de métodos observacionais. De facto, estudos futuros deverão conciliar diferentes métodos de análise, possibilitando um sistema integrado de informação quantitativa e qualitativa que potenciem a interpretação dos comportamentos individuais e coletivos.

Apesar do exposto, poder-se-á enfatizar a pertinência das presentes métricas para o conhecimento científico na análise de jogo. Comparativamente com outros métodos de análise (notacional e sequencial), as métricas de avaliação coletiva permitem uma identificação automática e exata da relação espáciotemporal entre jogadores, garantindo, assim, um instrumento de análise direta ou pós-jogo (dependente do tipo de tracking utilizado). No fundo, constitui-se como um potencial de informação célere e fiável de interpretação da relação entre jogadores ao longo do tempo.
Adicionalmente, a conciliação das métricas com indicadores diferenciadores (e.g., resultado do jogo, estado de posse de bola) possibilitam uma identificação específica das relações entre membros da equipa em função de constrangimentos distintos.

\section{CONCLUSÕES}

O presente trabalhou objetivou apresentar quatro métricas de avaliação coletiva que visam analisar a relação espacial entre jogadores de futebol da mesma equipa. As métricas coletivas delinearam-se para a identificação dos seguintes comportamentos: progressão coletiva; relação expansão-contração; e triangulações efetivas entre jogadores. Considerando os momentos com e sem posse de bola, foi possível verificar que as equipas na fase ofensiva avançam o seu centroid no campo, aproximando-se da baliza adversária. Foi igualmente possível verificar que as equipas aumentaram a sua dispersão nos momentos ofensivos e reduziram o espaço entre jogadores nos momentos sem posse de bola. Quanto à área efetiva de jogo foi possível verificar uma maior ocupação nos momentos com bola. Em suma, o presente trabalho demonstrou as potencialidades das métricas de avaliação coletiva para a análise de jogo no futebol, possibilitando um complemento à regular análise notacional e sequencial.

\section{IMPLICAÇÕES PRÁTICAS}

As métricas analisadas no presente artigo poderão servir como um contributo de informação para os técnicos desportivos, bem como, para o conhecimento científico na área de estudo da análise de jogo (Clemente et al., 2013). De facto, as limitações inerentes aos registos observacionais (e.g., análise notacional e modelos observacionais), apesar de reconhecidamente úteis e pertinentes para a análise de jogo, não possibilitam o reconhecimento automatizado e computorizado de relações espácio-temporais entre jogadores. Consequentemente, a adoção de métricas computacionais possibilitam um maior contributo das Ciências Exatas no campo das Ciências do Desporto, 

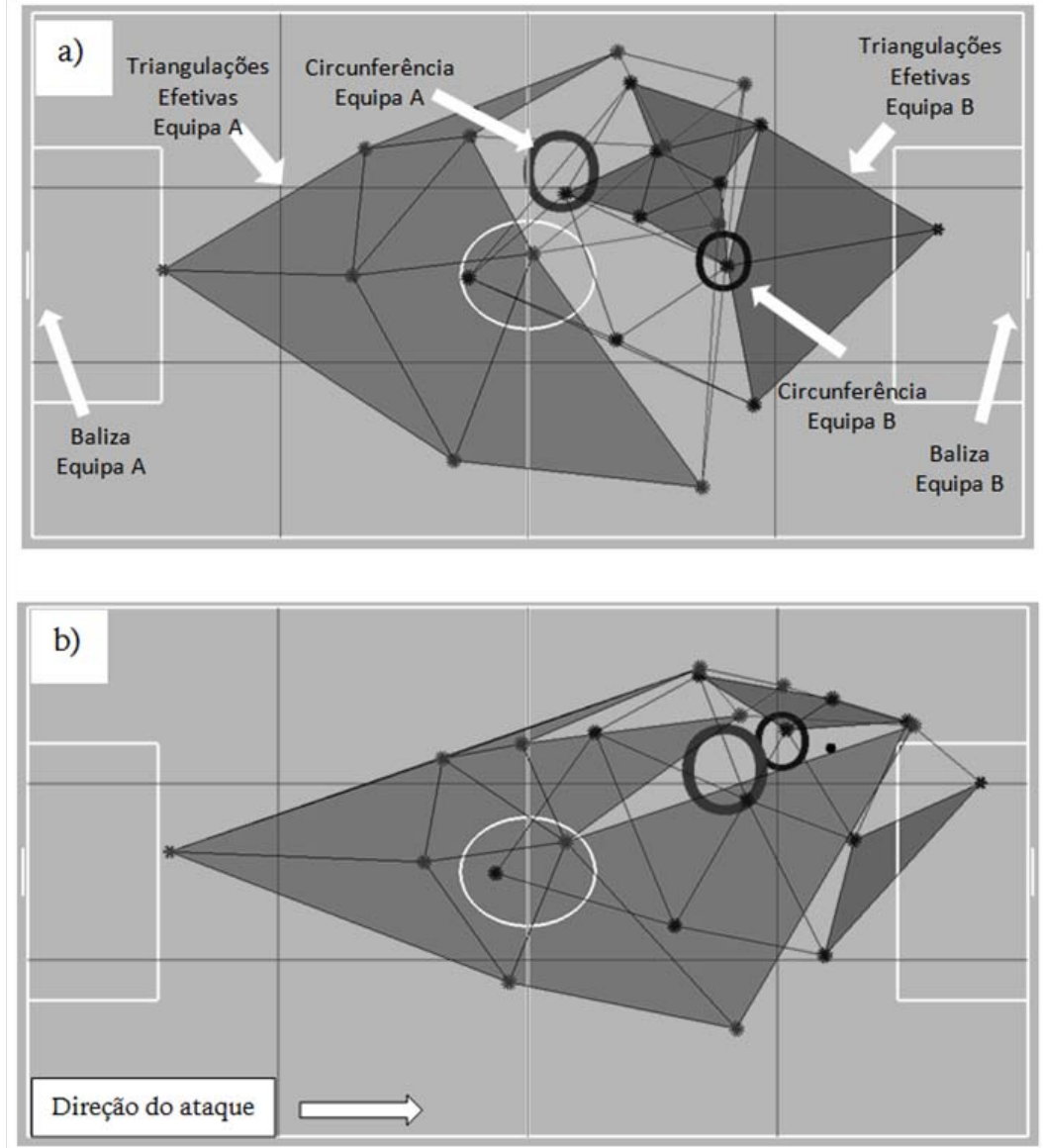

Figura 5. Duas situações de ataque da equipa que defende a baliza do lado esquerdo

conferindo-lhe um valor acrescentado no âmbito científico e aplicado.

Face à complexidade e potencialidade das métricas apresentadas neste artigo considerase pertinente apresentar alguns exemplos práticos expostos na Figura 5. Nesta figura, os triângulos não preenchidos representam as triangulações não-efetivas, os triângulos preenchidos com tonalidade cinza menos escura representam as triangulações efetivas da equipa A (nesta situação em posse de bola), sendo os mais escuros referentes à equipa $B$ (nesta situação sem posse de bola). Ainda na figura é possível verificar duas circunferências que representam individualmente o centroid ponderado e o índice dispersão ponderado sendo que, quanto maior a circunferência, maior será a dispersão da equipa. Quanto ao centroid ponderado é representado pelo ponto central da circunferência.
Uma possível interpretação das duas situações de jogo poderá relacionar-se imediatamente com o alinhamento e proximidade dos centroids ponderados (representados pelo ponto central das circunferências). De facto, é possível identificar que na situação a), o centroid ponderado da equipa B (a defender a baliza da direita na figura) encontra-se mais localizado no centro de terreno e não orientado longitudinalmente em função do centroid ponderado adversário (equipa A) com posse de bola. No entanto, existe um maior espaçamento (distância longitudinal) entre centroids ponderados, i.e., indicando que a equipa em fase ofensiva encontrar-se-ia em construção do momento ofensivo e com o bloco ofensivo recuado. $\mathrm{Na}$ situação b) os centroids ponderados encontramse deslocados para a região direita da equipa $B$ em fase defensiva. Neste caso, a proximidade dos centroids ponderados das equipas poder-se- 
á associar à maior proximidade entre os blocos ofensivo e defensivo. Ao longo do jogo, a existência de situações de ultrapassagem regular do centroid ponderado atacante sobre o defensivo poderá indiciar um nível elevado de perigo para a equipa em fase defensiva. De facto, estudos recentes indicam que esta situação de ultrapassagem do centroid ofensivo sobre o defensivo encontra-se significativamente relacionado com os golos sofridos em jogadas corridas (Frencken et al., 2011).

No que se refere à dispersão dos jogadores sobre o centroid ponderado é possível identificar a relação expansão-contração relatada na literatura (e.g., Moura et al., 2012). De facto, identifica-se graficamente o aumento da dispersão da equipa $\mathrm{A}$ em fase ofensiva relativamente à equipa em fase defensiva. No entanto, eventuais dispersões exageradas em situações defensivas poderão indiciar o incremento das possibilidades de penetração da equipa em posse de bola. Assim, o índice de dispersão ponderado possibilitará, para além da identificação da dispersão entre jogadores, a utilização de um identificador automático da dispersão fora de parâmetro normais (próprios da equipa). Este sistema de alerta informará os analistas sobre situações sucessivas de dispersão exagerada na fase defensiva que poderá comprometer a concentração defensiva e, por conseguinte, permitir penetrações ofensivas e exposição ao perigo.

Culminando a análise das implicações práticas das presentes métricas é possível identificar nas figuras 5 a) e b) que a equipa $B$ (em estado defensivo) reduz o número de triangulações defensivas efetivas na figura 5 b). Esta redução das triangulações defensivas efetivas (e consequentemente da área efetiva) indica um aumento do espaço entre jogadores que potenciará o aumento da exploração dos seus espaços intersectoriais por parte da equipa adversária com bola. É baseado no conceito da área efetiva (dependente das triangulações efetivas) que, à semelhança do proposto para o índice de dispersão, se poderão desenvolver sistemas automáticos de alarme, informando sobre a não constituição regular de triangulações efetivas, principalmente, entre os jogadores com maior preponderância na fase defensiva. Por exemplo, no caso da triangulação dos jogadores do meio-campo, a não proximidade entre os jogadores poderá resultar no não cumprimento de princípios de jogo como a cobertura defensiva, reduzindo as possibilidades de eficácia da intervenção defensiva. Assim, esta métrica possui o potencial de alertar os analistas para sucessivos afastamentos interpessoais que possam comprometer o sucesso do bloco defensivo.

A análise das implicações práticas procura não só justificar a pertinência e diferenciação das métricas propostas no presente artigo, mas também, enfatizar o potencial prático das mesmas no âmbito do treino desportivo. Utilizando tais ferramentas, serão possibilitados aos treinadores e analistas sistemas inteligentes que reconhecem padrões de comportamentos desviantes em função do comportamento tipo da própria equipa, alertando os observadores para eventuais necessidades de correção. Adicionalmente, o facto de serem sistemas que apenas dependem do rastreamento automático, poderão servir de plataformas online de trabalho bastando para tal conectar as informações das coordenadas posicionais dos jogadores com os algoritmos associados às métricas de avaliação coletiva. Esta informação automática poderá ser vital para uma maior celeridade na identificação de eventuais relacionamentos espáciotemporais entre jogadores, nomeadamente, para observadores com menor experiência na perceção de comportamentos coletivos.

Agradecimentos:

Nada a declarar.

Conflito de Interesses:

Nada a declarar.

Financiamento:

Nada a declarar. 


\section{REFERÊNCIAS}

Abdel-Aziz, Y., \& Karara, H. (1971). Direct linear transformation from comparator coordinates into object space coordinates. Comunicação apresentada no American Society of Photogrammetry Symposium on Close-Range Photogrammetry, Falls Church, Virginia, U.S.A..

Bartlett, R., Button, C., Robins, M., DuttMazumder, A., \& Kennedy, G. (2012). Analysing team coordination patterns from player movement trajectories in football: Methodological considerations. International Journal of Performance Analysis in Sport, 12 (2), 398-424.

Bourbousson, J., Sève, C., \& McGarry, T. (2010). Space-time coordination dynamics in basketball: Part 2 - The interaction between the two teams. Journal of Sports Sciences, 28(3), 349-358. doi: 10.1080/02640410903503640

Carling, C., Bloomfield, J., Nelsen, L., \& Reilly, T. (2008). The role of motion analysis in elite soccer: Contemporary performance measurement techniques and work rate data. Sports Medicine, 38(10), 839-862. doi: 10.2165/000 07256-200838100-00004

Carling, C., Williams, A. M., \& Reilly, T. (2005). Handbook of soccer match analysis: A systematic approach to improving performance. London \& New York: Taylor \& Francis Group.

Castelo, J. (1996). Futebol a organização do jogo: Como entender a organização dinâmica de uma equipa de futebol e a partir desta compreensão como melhorar o rendimento e a direç̧ão dos jogadores e da equipa. S.I.: Jorge Castelo.

Clemente, F. M., Couceiro, M. S., \& Martins, F. M. (2012). Towards a new method to analyze the soccer teams tactical behaviour: Measuring the effective area of play. Indian Journal of Science and Technology, 5(12), 3792-3801.

Clemente, F. M., Couceiro, M. S., Martins, F. M., \& Mendes, R. (2013). An Online Tactical Metrics Applied to Football Game. Research Journal of Applied Sciences, Engineering and Technology, 5(5), 1700-1719.

Costa, I. T., Garganta, J. M., Greco, P. J., \& Mesquita, I. (2009). Princípios táticos do jogo de Futebol: Conceitos e aplicação. Motriz, 15(3), 657-668.

Costa, I. T., Garganta, J., Greco, P. J., Mesquita, I., \& Seabra, A. (2010). Influence of relative age effects and quality of tactical behaviour in the performance of youth football players. Interna- tional Journal of Performance Analysis in Sport, 10(2), 82-97.

Davids, K., Button, C., \& Bennett, S. J. (2008). Dynamics of skill acquisition: A constraints-led approach. Champaign, Il: Human Kinetics.

Dooley, T., \& Titz, C. (2011). Football - The 4-4-2 System. Germany: Meyer \& Meyer Sport.

Duarte, R., Araújo, D., Correia, V., \& Davids, K. (2012). Sports teams as superorganisms: Implications of sociobiological models of behaviour for research and practice in team sports performance analysis. Sports Medicine, 42(8), 633-642. doi: 10.2165/11632450-00000000000000

Fernandes, O., Folgado, H., Duarte, R., \& Malta, P. (2010). Validation of the tool for applied and contextual time-series observation. International Journal of Sport Psychology, 41, 63-64.

Frencken, W., Lemmink, K., Delleman, N., \& Visscher, C. (2011). Oscillations of centroid position and surface area of football teams in smallsided games. European Journal of Sport Science, 11(4), 215-223. doi: 10.1080/17461391.2010. 499967

Gréhaigne, J. F., Godbout, P., \& Bouthier, D. (1999). The foundations of tactics and strategy in team sports. Journal of Teaching in Physical Education, 18, 159-174.

Hughes, M., \& Franks, I. M. (2004). Notational analysis: A review of the literature. In $\mathrm{M}$. Hughes \& I. M. Franks (Eds.), Notational analysis of sport: Systems for better coaching and performance in sport (pp. 59-106). New York: Routledge.

Lago, C. (2009). The influence of match location, quality of opposition, and match status on possession strategies in professional association football. Journal of Sports Sciences, 27(13), 14631469. doi: 10.1080/02640410903131681

Lago, C., \& Martín, R. (2007). Determinants of possession of the ball in soccer. Journal of Sports Sciences, 25(9), 969-974. doi: 10.1080/0264041 0600944626

Lago-Peñas, C., \& Dellal, A. (2010). Ball possession strategies in elite football according to the evolution of the match-score: The influence of situational variables. Journal of Human Kinetics, 25, 93-100. doi: 10.2478/v10078-010-0036-z

Lucchesi, M. (2001). Attacking Football: A Tactical Analysis. Auburn, Michigan: Reedswain Publishing. 
Maroco, J. (2010). Análise Estatística com utilização do SPSS. Lisboa: Edições Silabo.

Moura, F. A., Martins, L. E., Anido, R. O., Barros, R. M., \& Cunha, S. A. (2012). Quantitative analysis of Brazilian football players' organization on the pitch. Sports Biomechanics, 11(1), 85-96. doi: 10.1080/14763141.2011.637123

Pallant, J. (2011). SPSS Survival Manual: A Step by Step Guide to Data Analysis Using the SPSS Program. Australia: Allen \& Unwin.
Trapattoni, G. (1999). Coaching High Performance Football. Spring City, Pensylvania: Reedswain Inc.

Woltring, H. J., \& Huiskes, R. (1990) Stereophotogrammetry. In N. Berme \& A. Capozzo (Eds.), Biomechanics of Human Movement: Applications in rehabilitation, sports and ergonomics (pp. 108-127). Worthington, $\mathrm{OH}$ : Bertec Corporation. quando especificado em contrário e nos conteúdos retirados de outras fontes bibliográficas. 